\title{
Near-optimal Delay Constrained Routing in Virtual Circuit Networks
}

\author{
Hong-Hsu Yen ${ }^{1}$ and Frank Yeong-Sung Lin
}

\author{
Department of Information Management \\ National Taiwan University \\ Taipei, Taiwan, R.O.C. \\ Tel: $+886-2-23638423$ \\ Fax: $+886-2-27584773$ \\ Email: 14725001@im.ntu.edu.tw yslin@im.ntu.edu.tw
}

\begin{abstract}
An essential issue in designing, operating and managing a modern network is to assure end-to-end Quality-ofService (QoS) from users' perspective, and in the meantime to optimize a certain "average" performance objective from the system's perspective. In this paper, we consider the problem of minimizing the average cross-network packet delay in virtual circuit networks subject to an end-to-end delay constraint for each origin-destination user pair. The problem is formulated as a multicommodity network flow problem with integer routing decision variables, where additional end to end delay constraints are considered. The difficulties of this problem result from the integrality nature and particularly the nonconvexity associated with the end-to-end delay constraints. The basic approach to the algorithm development is Lagrangean relaxation in conjunction with a number of optimization-based heuristics. In the computational experiments, it is shown that the proposed algorithm calculates solutions which are within $1 \%$ and $3 \%$ of optimal solutions under lightly and heavily loaded conditions, respectively, in minutes of CPU time for networks with up to 26 nodes.
\end{abstract}

Index Terms - Optimization, Lagrangean Relaxation, End-toend QoS, Routing Assignment

\section{INTRODUCTION}

To ensure user-perceived end-to-end QoS requirement is one of the most important issues in providing modern network services, which typically requires sophisticated design of routing and capacity management policies. User-perceived end-to-end QoS measures include, for example, mean packet delay, packet delay jitter and packet lost probability. Besides users' perspective of QoS, from the service providers' perspective (which is a traditional view of network performance management), optimizing a certain system-level performance measure, e.g. overall network utilization or average cross-network delay among all users, is another major concern. Unfortunately, these two perspectives/objectives may not be entirely agreeable with each other. This then places a major challenge to network managers and therefore calls for an integrated methodology to consider these two perspectives in a joint fashion.

\footnotetext{
${ }^{1}$ Correspondence Author
}

The routing problem in virtual circuit networks has been a traditional research topic in computer networks and has attracted even more attention since the emergence of the Asynchronous Transfer Mode (ATM) technology. However, most previous research on virtual circuit routing considers the objective function of minimizing the average end-to-end packet delay $[1,3,5]$, which addresses a system-optimization perspective without taking individual users into account. In [2], Cheng and Lin took a user-optimization approach and considered a fairness issue by minimizing the maximum individual end-to-end packet delay in virtual circuit networks. In this paper, we attempt to jointly consider both perspectives. More precisely, we consider the virtual circuit routing problem of minimizing the average packet delay subject to end-to-end packet delay constraints for users. This problem is a difficult NP-complete problem as indicated in [6]. An optimizationbased approach is then devised to attack the problem, where the problem is formulated as a mathematical programming problem, followed by proposing an algorithm based on Lagrangean relaxation. It is shown in the computational experiments that the proposed algorithm is both efficient and effective.

The remainder of this paper is organized as follows. In Section 2, a mathematical formulation of the routing problem is proposed. In Section 3, a solution approach to the routing problem based on Lagrangean relaxation is presented. In Section 4, heuristics are developed to calculate good primal feasible solutions. In Section 5, computational results are reported. Finally, Section 6 concludes this paper.

\section{Problem Formulation}

The virtual circuit network is modeled as graph where the processors are depicted as nodes and the communication channels are depicted as arcs. We show the definition of the following notation.

\begin{tabular}{|c|l|}
\hline$V$ & $=\{1,2, \ldots, N\}$, the set of nodes in the graph \\
\hline$L$ & $\begin{array}{l}\text { the set of communication links in the communication } \\
\text { network }\end{array}$ \\
\hline$W$ & the set of Origin-Destination (O-D) pairs in the network \\
\hline
\end{tabular}




\begin{tabular}{|c|l|}
\hline$\gamma_{W}$ & $\begin{array}{l}\text { packets/sec): the arrival rate of new traffic for each O-D } \\
\text { pair } w \in W, \text { which is modeled as a Poisson process for } \\
\text { illustration purpose }\end{array}$ \\
\hline$C_{l}$ & (packets/sec), the capacity of each link $l \in L$ \\
\hline$P_{w}$ & $\begin{array}{l}\text { a given set of simple directed paths from the origin to the } \\
\text { destination of O-D pair } W\end{array}$ \\
\hline$x_{p}$ & $\begin{array}{l}\text { a routing decision variable which is } 1 \text { when path } p \in P_{W} \text { is } \\
\text { used to transmit the packets for O-D pair } W \text { and } \\
\text { otherwise }\end{array}$ \\
\hline$\delta_{p l}$ & $\begin{array}{l}\text { the indicator function which is } 1 \text { if link } 1 \text { is on path } p \text { and } \\
0 \text { otherwise }\end{array}$ \\
\hline$g_{l}$ & $\begin{array}{l}\text { the aggregate flow over link } l, \text { which is equal to } \\
\sum_{p \in P_{w}} \sum_{w \in W} x_{p} \gamma_{W} \delta_{p l}\end{array}$ \\
\hline$D_{w}$ & the maximum allowable end-to-end delay for O-D pair $W$ \\
\hline
\end{tabular}

Under the assumption of Kleinrock's independent assumption [4], the arrival of packets to each buffer is a Poisson process where the rate is the aggregate flow over the outbound link. Assume that the transmission time for each packet is exponentially distributed with mean $C^{-1}{ }_{k}$. Hence, refer to previous research $[1,3,5]$, each buffer is modeled as an $M / M / 1$ queue.

It is remarkable to address that the formulation can be extended to any non $M / M / 1$ model with monotonically increasing and convexity performance metrics. For the illustration purpose, the formulation will be based on the $M / M / 1$ model. To determine a path for each O-D pair to minimize the average packet delay with maximum allowable end-to-end transmission delay is formulated as a nonlinear combinatorial optimization problem, as shown below.

$$
Z_{I P^{\prime \prime}}=\min \frac{1}{\sum_{w \in W} \gamma_{W}} \sum_{l \in L} \frac{g_{1}}{C_{l}-g_{l}}
$$

subject to:

$$
\begin{array}{lr}
\sum_{l \in L} \sum_{p \in P_{w}} \frac{x_{p} \delta_{p l}}{C_{l}-g_{l}} \leq D_{W} & \forall W \in W \\
g_{l}=\sum_{p \in P_{w}} \sum_{w \in W} x_{p} \gamma_{W} \delta_{p l} \leq C_{l} & \forall l \in L \\
\sum_{p \in P_{w} x_{p}=1} & \forall W \in W \\
X_{p}=0 \text { or } 1 & \forall p \in P_{W}, W \in W .
\end{array}
$$

Constraint (1.1) requires that the end-to-end packet delay should be no larger than $D_{w}$ for each O-D pair. Constraint (1.2) requires that the aggregate flow on each link should not exceed the link capacity. Constraints (1.3) and (1.4) require that the all the traffic for each O-D pair should be transmitted over exactly one path. The above formulation is a nonlinear multicommodity flow problem, since each O-D pair transmits different type of traffic over the network. And it is easy to show that (IP") is a nonconvex programming problem by verifying the Hessian of $\sum_{l \in L} \sum_{p \in P_{w}} \frac{x_{p} \delta_{p l}}{C_{l}-g_{I}}$ with respect to $x_{p}$.
For the purpose of applying Lagrangean relaxation method, we transform the above problem formulation (IP") into an equivalent formulation (IP). In (IP), two auxiliary variables are introduced: $y_{w l}$ is defined as $\sum_{p \in P_{w}} x_{p} \delta_{p l}$ and $f_{l}$ denotes the estimate of the aggregate flow.

$$
Z_{I P}=\min \frac{1}{\sum_{w \in W} \gamma_{W}} \sum_{l \in L} \frac{f_{1}}{C_{l}-f_{l}}
$$

subject to:

$$
\begin{array}{lr}
\sum_{l \in L} \frac{y_{w l}}{C_{l}-f_{l}} \leq D_{w} & \forall w \in W \\
\sum_{p \in P_{w l}} x_{p}=1 & \forall w \in W \\
x_{p}=0 \text { or } 1 & \forall p \in P_{w}, W \in W \\
\sum_{p \in P_{w}} x_{p} \delta_{p l} \leq y_{w l} & \forall W \in W, l \in L \\
y_{w l}=0 \text { or } 1 & \forall W \in W, l \in L \\
g_{l} \leq f_{l} & \forall l \in L \\
0 \leq f_{l} \leq C_{l} & \forall l \in L .
\end{array}
$$$$
\forall l \in L \text {. (2.7) }
$$

Redundant constraints associated with these auxiliary variables from (2.4) to (2.7) are added. Note that Constraints (2.4) and (2.6) should be equalities, and it is clear that the equality should hold at the optimal point. By introducing these auxiliary variables, the Lagrangean relaxation problem can be decomposed into independent and easily solvable subproblems.

\section{LAGRANGEAN RELAXATION}

The algorithm development is based upon Lagrangean relaxation. We dualize Constraints (2.1), (2.4) and (2.6) to obtain the following Lagrangean relaxation problem (LR).

$$
\begin{gathered}
Z_{D}(t, u, v)=\min \left[\frac{1}{\sum_{w \in W} \gamma_{W}} \sum_{l \in L} \frac{f_{l}}{C_{l}-f_{l}}+\sum_{w \in W} t_{w}\left(\sum_{l \in L} \frac{y_{w l}}{C_{l}-f_{l}}-\right.\right. \\
\left.\left.D_{w}\right)+\sum_{w \in W} \sum_{l \in L} v_{w l}\left(\sum_{p \in P_{w}} x_{p} \delta_{p l}-y_{w l}\right)+\sum_{l \in L} u_{l}\left(g_{l}-f_{l}\right)\right]
\end{gathered}
$$

subject to:

$$
\begin{array}{lr}
\sum_{p \in P_{w}} x_{p}=1 & \forall W \in W \\
x_{p}=0 \text { or } 1 & \forall p \in P_{W}, W \in W \\
y_{w l}=0 \text { or } 1 & \forall W \in W, l \in L \\
0 \leq f_{l} \leq C_{l} & \forall l \in L
\end{array}
$$$$
\forall W \in W \quad(3.1)
$$$$
\forall p \in P_{w}, W \in W
$$

We can decompose (LR) into two independent subproblems. 
Subproblem 1: for $x_{p}$

$$
\min \sum_{w \in W} \sum_{p \in P_{w}} \sum_{l \in L}\left(v_{w l}+u_{l} \gamma_{W}\right) x_{p} \delta_{p l}
$$

subject to (3.1) and (3.2).

Subproblem 2: for $y_{w /}$ and $f_{l}$

$\min \left[\sum_{l \in L}\left(\frac{1}{\sum_{w \in W} \gamma_{w}} \frac{f_{l}}{C_{l}-f_{l}}+\frac{\sum_{w \in W} t_{w} y_{w l}}{C_{l}-f_{l}}-\sum_{w \in W} v_{w l} y_{w l}-u_{l} f_{l}\right)-\right.$

$$
\left.D_{w} \sum_{W \in W} t_{W}\right]
$$

subject to (3.3) and (3.4).

(SUB1) can be further decomposed into $|W|$ independent shortest path problem with nonnegative arc weights. It can be easily solved by the Dijkstra's algorithm. The $-D_{w} \sum_{w \in W} t_{w}$

tem in the objective function of (SUB2) can be dropped first and added back to the objective value since it will not affect the optimal solution of (SUB2). Then (SUB2) can be decomposed into $|L|$ independent subproblems. For each link $l \in L$

$\min \left[\frac{1}{\sum_{w \in W} \gamma_{w}} * \frac{f_{l}}{C_{l}-f_{l}}+\frac{\sum_{w \in W} t_{W} y_{w l}}{C_{l}-f_{l}}-u_{I} f_{I}-\sum_{w \in W} v_{w l} y_{w l}\right]$

subject to:

$y_{w l}=0$ or $1 \forall W \in W$ and $0 \leq f_{l} \leq C_{l}$.

(SUB2.1) is a complicated problem due to the coupling of $y_{w l}$ and $f_{l}$. On the other hand, the $\frac{1}{\sum_{w \in W} \gamma_{w}} * \frac{f_{l}}{C_{I}-f_{l}}$ term in the objective function of (SUB2.1) is a nonnegative and monotonically increasing function with respect to $f_{b}$, and it will not affect the optimal value of the following terms in the (SUB2.1). Therefore, the algorithm developed in [2] can be used to solve (SUB2.1). Hence, the algorithm to solve (SUB2.1) is as follows:

Step 1. Solve $\left(\frac{t_{w}}{C_{l}-f_{l}}-v_{w l}=0\right)$ for each O-D pair $w$, call them the break points of $f_{\text {r }}$.

Step 2. Sorting these break points and denoted as $f_{1}^{1}, f_{1}^{2}, \ldots$,

$$
f_{l}^{n}
$$

Step 3. At each interval, $f_{I}^{i} \leq f_{l} \leq f_{l}^{i+1}, y_{w}\left(f_{l}\right)$ is 1 if $\frac{t_{w}}{C_{l}-f_{l}}-v_{w l} \leq 0$ and is 0 otherwise.

Step 4. Within the interval, $f_{l}^{i} \leq f_{l} \leq f_{l}^{j+1}$, let $a_{l}$ be $\sum_{w \in W} t_{w} y_{w l}\left(f_{l}\right)$ and $b_{l}$ be $\sum_{w \in W} v_{w l} y_{w l}\left(f_{l}\right)$, then the

0-7803-7016-3/01/\$10.00 @2001 IEEE local minimal is either at the boundary point, $f_{I}^{i}$ or

$$
f_{l}^{i+1} \text {, or at point } f_{l}^{*}=C_{1}-\sqrt{\frac{a_{l}}{u_{l}}} \text {. }
$$

Step 5. The global minimum point can be found by comparing these local minimum points.

According to the algorithms proposed above, we could successfully solve the Lagrangean relaxation problem optimally. By using the weak Lagrangean duality theorem (for any given set of nonnegative multipliers, the optimal objective function value of the corresponding Lagrangean relaxation problem is a lower bound on the optimal objective function value of the primal problem), $Z_{D}(t, u, v)$ is a lower bound on $Z_{i p}$. We construct the following dual problem to calculate the tightest lower bound and solve the dual problem by using the subgradient method.

$$
Z_{D}=\max Z_{D}(t, u, v)
$$

subject to: $t, u, v \geq 0$.

Let the vector $S$ be a subgradient of $Z_{D}(t, u, v)$ at $(t, u, v)$. In iteration $x$ of the subgradient optimization procedure, the multiplier vector $m^{x}=\left(t^{x}, u^{x}, V^{x}\right)$ is updated by $m^{x+1}=m^{x}+\alpha^{x} S^{x}$, where $S^{x}(t, u, v)=\left(\sum_{l \in L} \frac{y_{w l}}{C_{l}-f_{l}}-D_{w}, g_{1}-f_{l}, \sum_{p \in P_{w}} x_{p} \delta_{p l}\right.$ $\left.-y_{w l}\right)$

The step size $\alpha^{x}$ is determined by $\delta \frac{Z_{I P}{ }^{h}-Z_{D}\left(m^{x}\right)}{\left\|S^{x}\right\|^{2}}$, where $Z_{I P}{ }^{h}$ is an primal objective function value (an upper bound on optimal primal objective function value), and $\delta$ is a constant $(0 \leq \delta \leq 2)$.

\section{Getting Primal Feasible Solutions}

To obtain the primal solutions to the minimized average packet delay with maximum allowable end-to-end delay constraints problems, solutions to the Lagrangean relaxation problems (LR) is considered. For example, if a solution to (LR) is also feasible to (IP), i.e., satisfy the capacity constraints and end-to-end delay constraints, then it is considered as a primal feasible solution to (IP); otherwise, it will be modified so that it may be feasible to (IP).

Three getting primal heuristics are developed to improve the effectiveness of the algorithms. For example, when a solution to (LR) is found, the routing assignments for the maximum end-to-end delay path is reassigned to another path to reduce the value of maximum end-to-end delay. For another case, consider that the end-to-end delay constraints, when the endto-end delay constraints is violated, identify the paths that violate end-to-end delay constraints, the arc weights along these paths are increased, then the routing assignments are recalculated. On the other hand, consider the capacity constraints, when a solution to (LR) is infeasible for capacity 
constraints, the arc weight for the overflow link is increased, and then the routing assignments are recalculated. According to the computational experiments, the second heuristic can get a better solution in many cases.

\section{COMPUTATIONAL EXPERIMENTS}

The computational experiments for the algorithms developed in Sections 3 and 4 are coded in $C$ and performed on a PC with INTEL ${ }^{\mathrm{TM}}$ PII-233 CPU. We tested the algorithm for 3 networks -- ARPA, GTE, OCT with 21, 12 and 26 nodes. The network topologies are shown in Fig. 1, 2 and 3.

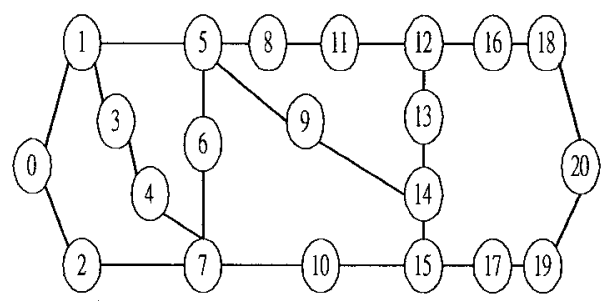

Figure 1. 21-node 52-link ARPA Network

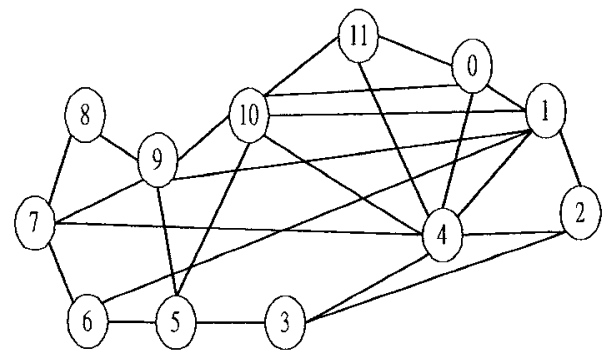

Figure 2. 12-node 50-link GTE Network

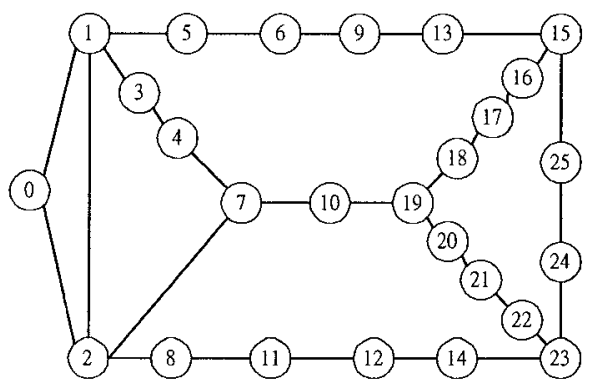

Figure 3. 26-node 60-link OCT Network

The maximum number of iterations for the proposed dual Lagrangean algorithm is 1000 , and the improvement counter is 30. The step size for the dual Lagrangean algorithm is initialized to be 2 and be halved of its value when the objective value of the dual algorithm, does not improve for 30 iterations. It is assumed that the traffic demand of each O-D pair is one packet per second. Unlike others' work in [2], the candidate path set does not need to be prepared in advance and all possible candidate paths are considered for each O-D pair.

We perform two sets of computational experiments. In the first set of computational experiments, the choice of the $D_{w}$ value is fixed as to examine the solution quality of the minimum average packet delay problem.

Table 1 summarizes the results. The first column is the type of the network topology. The second column is the link capacity. The third column is the maximum allowable end-toend delay $\left(D_{w}\right)$. The forth column reports the lower bound of the proposed dual Lagrangean problem. The fifth column reports the upper bound of the proposed dual algorithm. The sixth column reports the error gap between the lower bound and the upper bound. The seventh column reports the maximum end-to-end delay among all O-D pairs. As can be seen in the sixth column, the gap between the lower bound and the upper bound are very tight for all different network topologies and link capacities when the value of $D_{w}$ is loose as compared to the maximum end-to-end delay among all O-D pairs.

Since the value for the maximum allowable end-to-end delay $\left(D_{w}\right)$ have a significant impact on the solution of the minimum average packet delay problem. In the second set of computational experiments, we try to examine the impact of the $D_{w}$ value on the solution quality of minimum average delay. Fig. 4, 5 and 6 shows the results for the ARPA, GTE, OCT network. It is clear to see that the upper bound remains almost the same with different $D_{w}$ value. When the $D_{w}$ value below a certain threshold (as indicated in third column of Table 2), the primal solution could not be found.

Table 2 summarizes this result. The first column is the type of the network topology. The second column is the link capacity. The third column is the threshold of the maximum allowable end-to-end delay $\left(D_{w}\right)$. The forth column reports the lower bound of the proposed dual Lagrangean problem. The fifth column reports the upper bound of the proposed dual algorithm. The sixth column reports the error gap between the lower bound and the upper bound. The seventh column reports the maximum end-to-end delay among all O-D pairs. The eighth column reports the results from [2].

The maximum end-to-end delay value in the seventh column implies the optimal value for the minimax End-to-end Delay Routing problem developed in [2]. There is one thing that needs to be addressed: all possible candidate paths are considered for each O-D pair in this paper but only three candidate paths are pre-chosen for each O-D pair in [2]. Although we obtain a tighter upper bound than the minimax end-to-end delay routing problem developed in [2], this comparison is not on the same basis. On the other hand, the gap between the lower bound and the upper bound of the minimum average delay problem is still very tight, which indicate that the algorithms that we developed can achieve good system objective (average packet delay) even in stringent end-to-end delay requirements.

\section{CONCLUSIONS}

As compared to the work in [2], which tried to achieve better fairness among users by minimizing the maximum endto-end delay for virtual circuit networks without considering the system perspective (minimize the average packet delay). In this paper, for the first time, we considered the problem of minimizing the average packet delay with maximum allowable end-to-end delay requirements, which indicate that we try to obtain good system performance under user's end-to-end delay requirements.

We formulate this problem as a nonconvex and nonlinear 
multicommodity integral flow problem. The nonconvex and discrete (integer constraints) properties make the problem very difficult. We take an optimization-based approach by applying the Lagrangean relaxation technique in the algorithm development. According to the computational experiments, the error gap between the upper bound and the lower bound is so tight that we can claim that a near optimal solution is found. When the maximum end-to-end delay requirements are more and more close to the threshold, the upper bound (average packet delay) remains almost the same, this indicate that this solution approach can obtain good average packet delay solution under stringent end-to-end QoS requirements.

\section{REFERENCES}

[1] B. Gavish and S. L. Hantler, "An Algorithm for Optimal Route Selection in SNA Networks," IEEE Trans. on Comm., COM-31, pp. 1154-1160, Oct. 1983.

[2] K. T. Cheng and F. Y. S. Lin, "Minimax End-to-End Delay Routing and Capacity Assignment for Virtual Circuit Networks," Proc. IEEE Globecom, pp. 2134-2138, 1995.

[3] F. Y. S. Lin and J. R. Yee, "A New Multiplier Adjustment Procedure for the Distributed Computation of Routing Assignments in Virtual Circuit Data Networks," ORSA Journal on Computing, Vol. 4, No. 3, Summer 1992.

[4] L. Kleinrock, "Queueing Systems," Wiley-Interscience, New York, Vol. 1 and 2, 1975-1976.

[5] P. J. Courtois and P. Semal, "An Algorithm for the Optimization of Nonbifurcated Flows in Computer Communication Networks," Performance Evaluation, pp. 139-152, 1981.

[6] S. Chen and K. Nahrstedt, "An Overview of Quality of Service Routing for Next-Generation High-Speed Networks: Problems and Solutions," IEEE Network, pp. 64-79, Nov./Dec. 1998. 


\begin{tabular}{|c|c|c|c|c|c|c|}
\hline $\begin{array}{l}\text { Network } \\
\text { topology }\end{array}$ & $\begin{array}{c}\text { Link } \\
\text { capacity }\end{array}$ & $\overline{D_{w}(\mathrm{msec})}$ & Lower bound (msec) & Upper bound (msec) & Error gap (\%) & $\begin{array}{l}\text { Maximum end-to-end delay } \\
\text { (msec) }\end{array}$ \\
\hline ARPA & $\begin{array}{c}65 \\
70 \\
100 \\
150 \\
200\end{array}$ & $\begin{array}{l}460 \\
300 \\
250 \\
130 \\
100\end{array}$ & $\begin{array}{c}109 \\
93.7 \\
51.01 \\
29.084 \\
20.368\end{array}$ & $\begin{array}{c}110.3 \\
94.3 \\
51.06 \\
29.086 \\
20.3686\end{array}$ & $\begin{array}{c}1.18 \\
0.65 \\
0.1 \\
0.009 \\
0.003\end{array}$ & $\begin{array}{c}238.7 \\
200.7 \\
106.2 \\
60.4 \\
42.1\end{array}$ \\
\hline GTE & $\begin{array}{c}65 \\
70 \\
100 \\
150 \\
200\end{array}$ & $\begin{array}{l}800 \\
500 \\
100 \\
100 \\
190\end{array}$ & $\begin{array}{c}28.19 \\
26.02 \\
17.824 \\
11.687 \\
8.6936\end{array}$ & $\begin{array}{c}28.2 \\
26.04 \\
17.829 \\
11.688 \\
8.694\end{array}$ & $\begin{array}{c}0.05 \\
0.05 \\
0.03 \\
0.01 \\
0.005\end{array}$ & $\begin{array}{l}50.9 \\
46.9 \\
31.9 \\
20.8 \\
15.5\end{array}$ \\
\hline OCT & $\begin{array}{c}65 \\
70 \\
100 \\
150 \\
200\end{array}$ & $\begin{array}{l}1400 \\
1000 \\
340 \\
150 \\
100\end{array}$ & \begin{tabular}{|c|}
351.3 \\
237.8 \\
86.2 \\
42.42 \\
28.1529
\end{tabular} & $\begin{array}{c}357.8 \\
240.6 \\
86.8 \\
42.44 \\
28.1559\end{array}$ & $\begin{array}{l}1.88 \\
1.2 \\
0.69 \\
0.04 \\
0.01\end{array}$ & $\begin{array}{c}805 \\
525.6 \\
170.4 \\
81.7 \\
53.8\end{array}$ \\
\hline
\end{tabular}

TABLE 2 - Comparison Of Solution Qualtry ObTained By Various Networks At THE Threshold Of MaXimum Allowable END-To-End DelaY

\begin{tabular}{|c|c|c|c|c|c|c|c|}
\hline \multicolumn{8}{|c|}{ REQUIREMENTS } \\
\hline $\begin{array}{l}\text { Network } \\
\text { topology }\end{array}$ & $\begin{array}{c}\text { Link } \\
\text { capacity }\end{array}$ & $\begin{array}{c}\text { Threshold of } D_{w} \\
(\mathrm{msec})\end{array}$ & $\begin{array}{c}\text { Lower bound } \\
(\mathrm{msec})\end{array}$ & $\begin{array}{c}\text { Upper bound } \\
\text { (msec) }\end{array}$ & Error gap (\%) & $\begin{array}{l}\text { Maximum end-to-end } \\
\text { delay (msec) }\end{array}$ & Results from [2] \\
\hline ARPA & $\begin{array}{c}65 \\
70 \\
100 \\
150 \\
200 \\
\end{array}$ & $\begin{array}{c}237.7 \\
203 \\
110 \\
61 \\
43\end{array}$ & $\begin{array}{c}108.9 \\
93.6 \\
50.7 \\
28.97 \\
20.35\end{array}$ & $\begin{array}{c}10.3 \\
94.4 \\
51.2 \\
29.1 \\
20.37\end{array}$ & $\begin{array}{c}1.23 \\
0.9 \\
1.02 \\
0.5 \\
0.09\end{array}$ & $\begin{array}{c}237.2 \\
202.2 \\
108 \\
60.9 \\
42.3\end{array}$ & $\begin{array}{l}\text { N/A }{ }^{\dagger} \\
\text { N/A } \\
\text { N/A } \\
\text { N/A } \\
\text { N/A }\end{array}$ \\
\hline GTE & \begin{tabular}{|c|}
65 \\
70 \\
100 \\
150 \\
200 \\
\end{tabular} & $\begin{array}{c}50.88 \\
47 \\
32 \\
21.5 \\
15.5\end{array}$ & $\begin{array}{c}28.17 \\
26.026 \\
17.82 \\
11.68 \\
8.6 \\
\end{array}$ & $\begin{array}{c}28.23 \\
26.046 \\
17.83 \\
11.689 \\
8.7 \\
\end{array}$ & $\begin{array}{c}0.2 \\
0.08 \\
0.08 \\
0.07 \\
0.8 \\
\end{array}$ & $\begin{array}{c}50.876 \\
46.9 \\
31.9 \\
20.8 \\
15.49 \\
\end{array}$ & $\begin{array}{l}\text { N/A } \\
\text { N/A } \\
\text { N/A } \\
\text { N/A } \\
\text { N/A } \\
\end{array}$ \\
\hline OCT & $\begin{array}{c}65 \\
70 \\
100 \\
150 \\
200\end{array}$ & $\begin{array}{c}727 \\
470 \\
166.8 \\
81.2 \\
54\end{array}$ & $\begin{array}{c}351.3 \\
237.9 \\
86.24 \\
42.4 \\
28.04\end{array}$ & $\begin{array}{c}364.2 \\
240.8 \\
86.9 \\
42.5 \\
28.16\end{array}$ & $\begin{array}{c}3.67 \\
1.21 \\
0.8 \\
0.2 \\
0.4\end{array}$ & $\begin{array}{c}722 \\
469.8 \\
166.7 \\
81.1 \\
53.9\end{array}$ & $\begin{array}{c}860.3 \\
514.6 \\
168.4 \\
81.7 \\
54.1\end{array}$ \\
\hline
\end{tabular}

t: The work in [2] did not perform the computational experiments in these network settings.

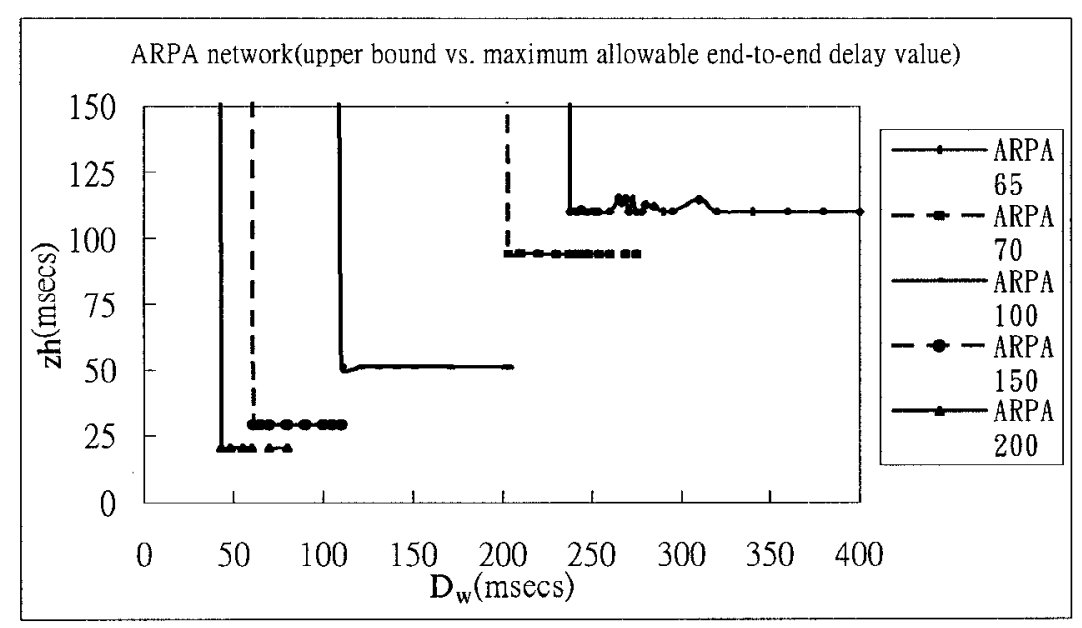

Figure 4. Upper bound for different $D_{w}$ value in ARPA network 


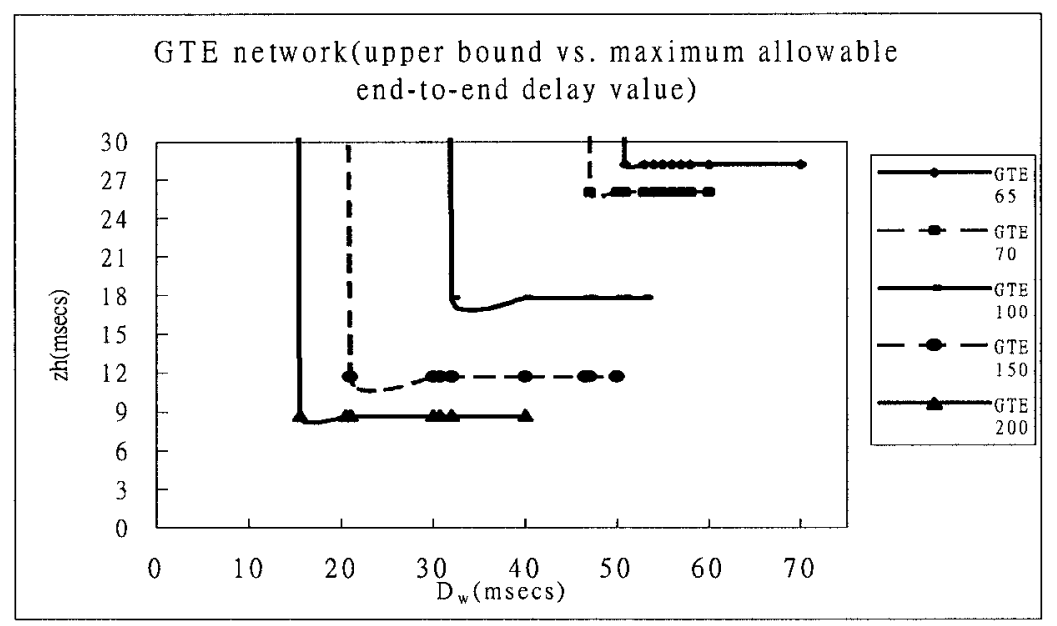

Figure 5. Upper bound for different $D_{w}$ value in GTE network

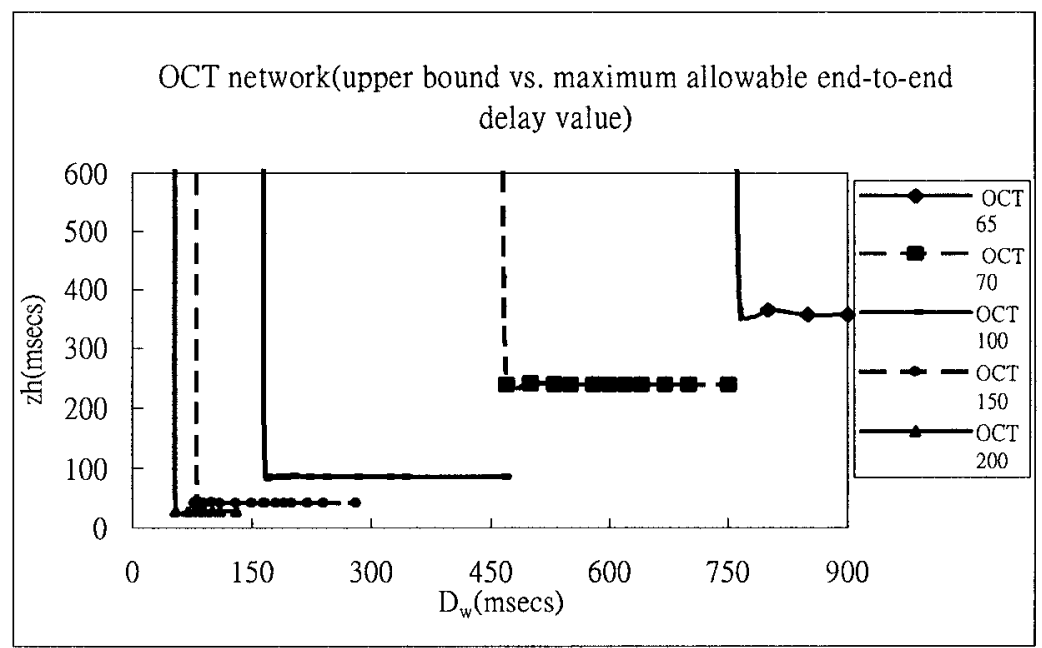

Figure 6. Upper bound for different $D_{w}$ value in OCT network 